\title{
science \\ The 5th Asian Science Editors' Conference and Workshop 2018
}

\author{
Suhyeon Yoo \\ Open Access Center, Korea Institute of Science and Technology Information, Seoul, Korea
}

Date: July 18-19, 2018

Venue: IPB International Convention Center, Bogor, Indonesia

URL: https://www.asianeditor.org/event/2018/

The Council of Asian Science Editors (CASE), a non-profit organization, was established in 2014 to improve the quality of scientific journals published in Asia through consulting and exchange of information on editing and publishing [1]. Since the inaugural "Asian Science Editors' Conference and Workshop" in 2014 in Seoul, CASE has held international conferences in major cities of Asia every year. The 5th CASE conference was held jointly with the Asia Pacific Association of Medical Journal Editors on July 18 to 19, 2018 at the IPB International Convention Center in Bogor, Indonesia. The conference topic was 'Digital standards and editors' associations of Asia-Pacific scientific journals' and over 270 participants from 22 countries attended. There were 44 presentations and six poster presentations.

I am a senior researcher at the Korea Institute of Science and Technology Information. Since this April I have served as a managing editor for the Journal of Information Science Theory and Practice (JISTaP), an international journal in the field of information science. I attended the conference to promote JISTaP, network with science editors, and examine international trends in the scholarly publishing community.

Through her interesting presentation, 'What manuscript editors do,' Jae Hwa Chang explained that a manuscript protocol that reflects the characteristics of individual journals should be developed. As a manuscript editor, she presented actual cases of editing in journals. Now, six years after the first issue of JISTaP, it is time for JISTaP to reorganize its manuscript guidelines. I have realized that it is necessary to establish JISTaP's own consistent and detailed bibliographic principles while essentially following the APA (American Psychological Association)

Received: July 25, 2018 Accepted: July 30, 2018

Correspondence to Suhyeon Yoo yoosu@kisti.re.kr

ORCID

Suhyeon Yoo

http://orcid.org/0000-0002-7541-6989 style. The most impressive presentation at the conference was 'New content formats and new channels: communicating science effectively beyond the journal article' by Basil D'souza from Editage. He presented examples of video, audio, and lay summaries while explaining that the articles' new content format opens the door to new possibilities. D'souza also mentioned that the research news story as one of the research communication services would improve communication between journal editors and authors. I wondered if such service could be applied in traditional academic journals, and whether this service would enable young researchers to 
access articles easily. On the other hand, I reflected on the role and scope of a journal editor, since I had to consider the content type and research communication service.

Through this brief but meaningful conference, I learned the following lessons as a newcomer to the scholarly publishing community, a researcher in the field of information science, and a journal managing editor.

The following summarizes what I learned as a newcomer to the scholarly publishing community. I obtained a general overview of publishing trends in Asian countries through eight countries' individual reports. Each country's report was presented for 10 minutes. In particular, Indonesia has developed a journal indexing system called SINTA to provide information in real time, and it has expanded and developed it into an international evaluation system. Additionally, I networked with attendees from Wordvice, Naver, Editage, and Aries Systems to understand the perspectives of each stakeholder in the scholarly publishing community.

Second, as an information service researcher, I gained the following insights. The conference covered various topics, with four plenary lectures, six workshops, three sessions, and four group meetings. In some cases, similar topics were presented, so I wondered how they related to presentations from previous conferences. Compared to the fourth international conference, which featured 12 sessions and 200 attendees [2], the conference's scale increased in 2018. Presentations in diverse fields such as publishing, government, and non-profit organizations were offered. Thus, I learned that the scope of science editing has expanded.

I was also inspired to think up some new research ideas. First, as the academic and practical scope of the science editing field has expanded, it is necessary to set a specific subject category. If the subject category were defined, it would be possible to hold a discussion based on each topic and field and to collect relevant data in one place. In addition, this would enable session organization according to predetermined subject categories and networking between people with the same thematic interests in future conferences. Second, there needs to create a repository for the field of science editing. Given the potential difficulty of building a repository in a short period of time due to technical, financial, and policy issues, they may start by simply collecting data from the CASE website. In other words, the content presented at the conference could be archived according to subject category and related topic articles from science editing journals. Eventually, researchers may derive a new research topic or determine their conference presentation topics based on data in the corresponding subject category. I will extract and group journal keywords related to scholarly publishing and editing-such as Science Editing, Learned Publishing, Science Editor, European Science Editing,

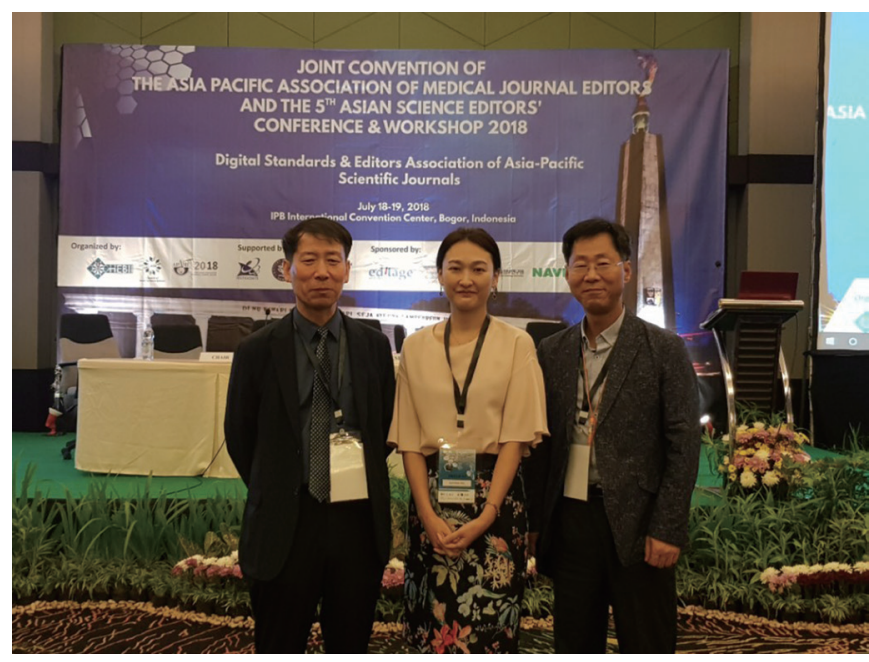

Fig. 1. Journal of Information Science Theory and Practice (JISTaP) management team. From left, Dong-Geun Oh (JISTaP, co-Editor in Chief), Suhyeon Yoo (JISTaP, managing editor), and Tae-Sul Seo (Director of Open Access Center of Korea Institute of Science and Technology Information).

and Journal of Scholarly Publishing-to derive major topics. I will forward the extracted topics to the CASE Executive Office to help schedule the next CASE conference. Moreover, I had the idea to define terms related to scholarly publishing before developing the subject category and repository. Collecting and organizing the terms used by various stakeholders to create a glossary is a basic prerequisite for smooth communication among people engaged in the scholarly publishing community, which is an attractive and extensive field.

Third, as a managing editor who publishes journals, I learned the following lessons. I managed a JISTaP promotion booth for two days, which attracted many people's attention. Therefore, it was a good opportunity to publicize Korea Institute of Science and Technology Information, a journal publisher as well as information research institute of Korea, and the journal itself (Fig. 1). JISTaP was listed on the Scopus in 2017, but one attendee asked me about the journal's SCImago Journal \& Country Rank [3]. Thus, it became evident that while Koreans evaluate journals based on Scopus listings, foreigners evaluate journals based not only on Scopus but also on SJR listings. Next to our booth, Wordvice-an English editing and proofreading company-operated a promotion booth. This company provides English proofreading and editing services and offers various video lectures and articles to help researchers write their manuscripts effectively and efficiently. Wordvice has various resources such as abstract writing tips, a cheat sheet of useful phrases for academic papers, and an article on five common reasons manuscripts are rejected [4]. These will be useful for researchers who submit papers to our journal if hyperlinked on our journal website. 
What will I learn at the next conference? How will I be inspired by other people's interests and ideas? I believe that the academic and practical scope of the science editing field will become deeper and wider at the next conference.

\section{Conflict of Interest}

No potential conflict of interest relevant to this article was reported.

\section{References}

1. Council of Asian Science Editors. History [Internet]. Seoul: Council of Asian Science Editors [cited 2018 Aug 1]. Available from: https://www.asianeditor.org/about/history.php

2. Kim S. The 4th Asian Science Editors' Conference and Workshop 2017. Sci Ed 2017;4:101-4. https://doi.org/10.6087/kcse.106

3. SCImago Journal \& Countury Rank. About us [Internet]. Amsterdam: Elsevier [cited 2018 Aug 1]. Available from: https://www.scimagojr.com/aboutus.php

4. Wordvice. Academic resources [Internet]. Seongnam (KR): Wordvice [cited 2018 Aug 1]. Available from: https://wordvice.com/academy-academic/ 